\title{
Determining 2D temperature field in flow boiling with the use of Trefftz functions
}

\author{
Sylwia Hoüejowska ${ }^{*}$ \\ Faculty of Management and Computer Modelling, Kielce University of Technology, Al. 1000-lecia P.P. 7, 25-314 Kielce, Poland
}

\begin{abstract}
The paper proposes the use of Trefftz method to solve the triple coupled heat conduction problem in flow boiling of refrigerant in an asymmetrically heated minichannel. A mathematical model of heat transfer in a rectangular minichannel is suggested. Two sets of Trefftz functions were used to determine 2D temperature fields at a fluid flow in the minichannel tilted at a known angle. The procedure for the calculation of the liquid temperature was coupled with the process of determining temperature fields in two adjacent elements of the experimental stand with the minichannel, i.e. in the glass pane and the heating foil. Heat transfer in the glass, foil and liquid is described using various 2D differential equations with an adequate set of boundary conditions. Solving those equations led to the solving of the triple coupled heat conduction problem made up of one direct and two subsequent inverse problems. The results are presented as: (1) 2D temperature of the glass pane, the heating foil, the flowing liquid, (2) mean square errors between temperature approximations and selected boundary conditions, (3) the heat transfer coefficient versus the distance from the minichannel inlet.
\end{abstract}

\section{Introduction}

Progress in the miniaturization of modern components and systems, rendered possible by technological advances, has led to the development of methods ensuring their functionality and proper performance. Reduced energy intensity and material consumption are required from todayôs tiny heat exchangers, while maintaining or possibly improving thermodynamic performance by carrying increased heat fluxes. One of the ways to intensify heat transfer and satisfy the criteria above is associated with the phase change of refrigerants, in particular in flow boiling in minichannels. This creates the idea of the so-called compact heat exchangers (evaporators and condensers) within cooling systems. Owing to small internal volumes, the minichannels additionally satisfy the environmental criterion, as in the case of a leaking system, the amount of the refrigerant carried to the surroundings will be negligible. Proper operation of high-efficiency miniature heat exchangers depends on strictly specified and stable flow. The designing process faces the problem of the selection of suitable calculation procedures (especially for the inverse heat conduction problems, IHCP) that will allow the identification of processes governing heat transfer in the small-sized channels.

The paper presents an application of the Trefftz method for solving stationary problems of heat exchange in minichannels. The Trefftz method, belonging to the meshless methods group, is an effective method which allows for obtaining stable IHCP solutions [1-3]. The method has been used successfully for determining nonstationary IHCP solutions too [4]. A hybrid combination of the Trefftz method with other numerical methods, e.g. the Picard method or the homotopy perturbation method, also allows for solving non-linear IHCPs, including nonstationary ones [5-7]. The idea of the Trefftz method consists in an approximation of an unknown solution of a linear partial differential equation by a linear combination of functions satisfying this governing equation exactly. These functions are called Trefftz functions. The linear combination coefficients are determined base on known boundary conditions.

In this paper the Trefftz method was used to solve a triple coupled heat conduction problem made up of one direct and two subsequent inverse problems. In the mathematical model, the heat exchange process in the elements of an experimental stand, i.e. an insulating glass pane, a heating foil and a minichannel was described using the Laplaceôs equation, the Poissonôs equation, and the energy equation, respectively. The boundary conditions reported for each of the equations led to the solution of a triple coupled heat conduction problem made up of one direct (in the glass) and two subsequent inverse problems (in the foil and the liquid). Two dimensional temperature distributions in the glass pane and the heating foil were determined using harmonic polynomials [1]. To model the temperature field in the third region $\ddot{i}$ the liquid $\ddot{i}$ the Trefftz functions given for the energy equation were used [3], at the velocity profile adopted to take into account the minichannel tilt angle. 


\section{Description of the experiment}

The experimental section, described here in brief, has been presented in more detail in [8, 9]. In this experiment, with little difference between the temperatures of the heating surface and the liquid, heat transfer is enhanced through the phase change during boiling. When a enhanced heating surface is used in the minichannel on the side of the liquid, the heat transfer process is further intensified [10].

Figure 1 shows the test module tilted at a $\omega$ angle, with a rectangular minichannel and FC-72 flowing through it.

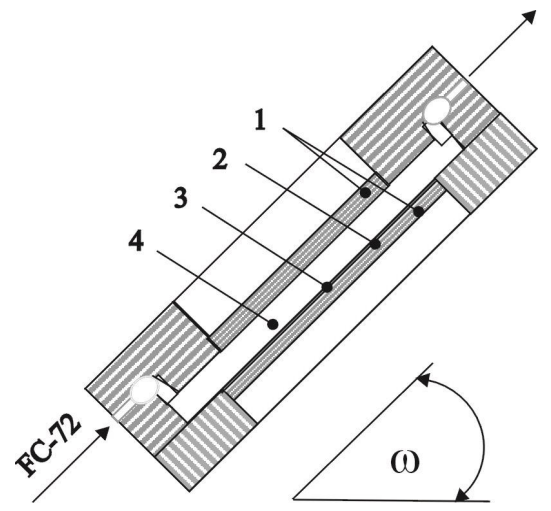

Fig.1. Test module: 1- separating glass panes, 2- heating foil, 3- a layer of liquid crystals, 4- minichannel (illustrative figure, not to scale).

One of the minichannel walls is made of the heating foil with a modified surface, supplied with a current of steady intensity. Two glass plates on opposite sides of the minichannel separate it from the space of the surrounding unit. Flow structures can be observed through one of the plates. A layer of liquid crystals covering the foil from the side of the glass plate allows obtaining a 2D temperature field of the heating surface. The measurements include: local foil temperature values at the foil-glass interface, the temperature and pressure of the refrigerant at the inlet and outlet of the minichannel, current and voltage drop of the electric power supplied to the foil, the mass flux $[8,9]$.

\section{Mathematical model and method}

The mathematical model presented in the paper is a modification of the approach reported in $[3,7]$. In the model of the heat transfer problem occurring during the flow of FC-72 refrigerant through the minichannel, the assumptions included the steady state in the test module and slight temperature changes of the glass, foil and the liquid along the minichannel width. The proposed approach considered two dimensions: dimension $x$ in the direction of the flow and dimension $y$ perpendicular to the flow, related to the thickness of the glass plate $\left(\delta_{G}\right)$ and heating foil $\left(\delta_{F}\right)$, and to the depth of the minichannel $\left(\delta_{M}\right)$. The layer of liquid crystals, very thin, was skipped in the model. Further considerations refer only to the central section of the measurement module. In the notation used, the parameters for the glass plate, heating foil and liquid are indicated by subscripts: $G$ for the glass partition, $F$ for the heating foil , $L$ for the liquid and $M$ for minichannel.

The protecting glass temperature $T_{G}$ and the heating foil $T_{F}$ satisfied the Laplace equation and the Poisson equation, respectively [3]

$$
\begin{aligned}
& \nabla^{2} T_{G}=0,(x, y) \in \Omega_{G} \\
& \nabla^{2} T_{F}=-\frac{q_{V}}{\lambda_{F}},(x, y) \in \Omega_{F}
\end{aligned}
$$

where $\Omega_{G}=\left\{(x, y) \in R^{2}: 0<x<H, \quad 0<y<\delta_{G}\right\}$ is the glass temperature variation domain, $\Omega_{F}=\left\{(x, y) \in R^{2}: 0<x<H, \quad \delta_{G}<y<\delta_{G}+\delta_{F}\right\} \quad$ is the heating foil temperature variation domain, $H$ - height of the minichannel, $q_{V} \ddot{i}$ volumetric heat flux supplied to the foil, $\lambda$ - thermal conductivity and $\nabla^{2}=\frac{\partial^{2}}{\partial x^{2}}+\frac{\partial^{2}}{\partial y^{2}}$.

At the glass $\ddot{i}$ foil interface the temperatures and heat fluxes were assumed to match, i.e.

$$
\begin{gathered}
T_{G}\left(x_{m}, \delta_{G}\right)=T_{F}\left(x_{m}, \delta_{G}\right)=T_{m} \text { for } m=1,2, \ldots, M \\
\lambda_{F} \frac{\partial T_{F}}{\partial y}=\lambda_{G} \frac{\partial T_{G}}{\partial y} \text { for } y=\delta_{G}, 0 \leq x \leq H
\end{gathered}
$$

where $T_{m}$ stands for the discrete temperature measurements made at measurement points $\left(x_{m}, \delta_{G}\right)$.

The remaining boundaries were assumed to be isolated, excluding the edges of the foil in contact with the liquid, [3, 6].

The theoretical model of heat transfer in the liquid assumed that the liquid temperature in domain $\Omega_{L}=\left\{(x, y) \in R^{2}: 0<x<H, \quad \delta_{G}+\delta_{F}<y<\delta_{G}+\delta_{F}+\delta_{M}\right\}$ fulfill the energy equation in the form

$$
\begin{aligned}
& \lambda_{L} \Delta T_{L}=w_{x}(y) c_{p} \rho_{L} \frac{\partial T_{L}}{\partial x}-\mu_{L} \Phi+ \\
& -w_{x}(y) \frac{d p}{d x}-\rho_{L} g w_{x}(y) \sin \omega
\end{aligned}
$$

where the function $\Phi=\left(\frac{d w_{x}}{d y}\right)^{2}$ was the Rayleigh dissipation function, $\frac{d p}{d x} \approx \frac{\Delta p}{H}, w_{x^{-}}$velocity, $\Delta p$ - pressure drop, $c_{p}, \rho_{L}, \mu_{L}$ - specific heat, density and dynamic viscosity of the liquid, respectively, $g$ - standard gravity.

The following boundary conditions were adopted for equation $(5),[3,6,7]$ :

$i$ the liquid flow in the minichannel is laminar ( $\operatorname{Re}<2000)$ and stationary with a fixed mass flux density,

$i$ the resultant two-phase mixture has void fraction $\varphi(x)$ constant in the given cross-section of the minichannel,

$i \quad$ the velocity of the liquid flowing in the minichannel has only one non-zero component $w_{x}=w_{x}(y)$, parallel to 
the heating surface and flow direction, satisfying the condition from the momentum equation

$$
-\rho_{L} g \sin \omega-\frac{d p}{d x}+\mu_{L} \frac{\partial^{2} w_{x}}{\partial y^{2}}=0
$$

i the liquid temperature at the inlet $\left(T_{i n}\right)$ and outlet $\left(T_{\text {out }}\right)$ of the minichannel is known,

$\ddot{i}$ the liquid temperature at the contact with the foil fulfills the condition

$$
T_{L}= \begin{cases}T_{F}, & \text { if } T_{F}<T_{\text {sat }} \\ T_{s a t}, & \text { if } T_{F} \geq T_{\text {sat }}\end{cases}
$$

where $T_{\text {sat }}$ means the saturation temperature,

$\ddot{i}$ for $y=\delta_{G}+\delta_{F}, 0 \leq x \leq H$ the whole heat flux generated in the heating foil is transferred to the liquid phase in the proportion resulting from the void fraction

$$
\lambda_{L} \frac{\partial T_{L}}{\partial y}=\lambda_{F}(1-\varphi(x)) \frac{\partial T_{F}}{\partial y}
$$

where by the void fraction was calculated based on the homogeneous model [11]

$$
\varphi(x)=\left(1+\left(\frac{1-X(x)}{X(x)}\right)\left(\frac{\rho_{v}}{\rho_{L}}\right)\right)^{-1}
$$

The thermodynamic vapor quality $X(x)$ was calculated from [12]

$$
X(x)=\frac{q_{V} \delta_{F} H\left(x-x_{s a t}\right)}{A G h_{L v}}
$$

where $A$ - field of the heating surface, $G$ - mass flux, $h_{L v^{-}}$ enthalpy, $x$ is the distance from the heated section starting point, $x_{\text {sat }}$ - the location where boiling starts determined formula, [3]

$$
x_{s a t}=\frac{G A_{M} c_{p}\left(T_{s a t}-T_{i n}\right)}{q_{V} \delta_{F} H}
$$

Where the heating foil temperature distribution and the temperature gradient were known, the heat transfer coefficient $\alpha(x)$ at the foil-liquid interface could be determined using the Robin condition

$$
\alpha(x)=\frac{-\lambda_{F} \frac{\partial T_{F}}{\partial y}\left(x, \delta_{G}+\delta_{F}\right)}{T_{F}\left(x, \delta_{G}+\delta_{F}\right)-T_{a v e}(x)}
$$

In (12) the reference temperature $T_{\text {ave }}$ was taken as the average temperature at points from $x=0$ to $x=0.5 \delta_{M}$ (eq. 13a) or along the thickness of the thermal layer $\delta_{T}$ (13b)

$$
\text { a) } T_{a v e, 1}(x)=\frac{1}{0.5 \delta_{M}} \int_{\delta_{G}+\delta_{F}}^{\delta_{G}+\delta_{F}+0.5 \delta_{M}} T_{L}(x, y) d y
$$

$$
\text { b) } T_{a v e, 2}(x)=\frac{1}{\delta_{T}} \int_{\delta_{G}+\delta_{F}}^{\delta_{G}+\delta_{F}+\delta_{T}} T_{L}(x, y) d y
$$

The thermal layer $\delta_{T}$ was calculated according to $[7,13$, 14].

\subsection{Trefftz method}

The unknown approximations of the glass, foil and liquid temperature were determined using the Trefftz method. First, the approximation of the glass partition temperature $T_{G}$ was found by solving the direct problem in the procedure described in [15]. Then, the approximation of the heating foil temperature $T_{F}$ was determined by solving the first IHCP. These approximations were calculated using the harmonic polynomials (the Trefftz functions for the Laplace equation). Finally, the approximation of the liquid temperature $T_{L}$ was determined by solving the next IHCP. The Trefftz functions for the energy equation in the form

$$
\lambda \Delta T=w_{x}(y) c_{p} \rho \frac{\partial T}{\partial x}
$$

were presented in [16]. In [17] the properties of these functions and their relationship to harmonic polynomials were described. In this case the unknown temperature $T_{L}$ was written in the form

$$
T_{L}(x, y)=T_{s o l}(x, y)+\sum_{n=0}^{N_{L}} a_{n} \theta_{n}(x, y)
$$

where $T_{s o l}(x, y)$ is the particular solution to equation (5).

The coefficients $a_{n}$ of the linear combination (15) were computed by minimizing the error functional which describes the mean square error with which the liquid temperature approximation $T_{L}$ satisfies the adopted boundary conditions [3, 16]. Determined using the Trefftz functions, the temperature approximations for the glass plate $T_{G}$, heating foil $T_{F}$ and liquid $T_{L}$ are continuous functions that satisfy equations (1), (2) and (5) exactly and the boundary conditions adopted for these equations approximately.

\section{Results}

The numerical calculations were made for two sets of data from the experiment for the forced flow of FC-72 refrigerant through the asymmetrically heated minichannel, tilted at angle $\omega=45^{\circ}$, with dimensions: $0.36 \mathrm{~m}$ in length, $0.001 \mathrm{~m}$ in depth and $0.04 \mathrm{~m}$ in width. Other basic experimental parameters included the Reynolds number, $R e=750$, the mass flux, $205 \mathrm{~kg} /\left(\mathrm{m}^{2} \mathrm{~s}\right)$, pressure at the inlet to the minichannel, $142 \mathrm{kPa}$, the average liquid velocity in the minichannel, $w_{\text {ave }}=0.12 \mathrm{~m} / \mathrm{s}$, liquid temperature at the minichannel inlet, $296 \mathrm{~K},[8,9]$. 

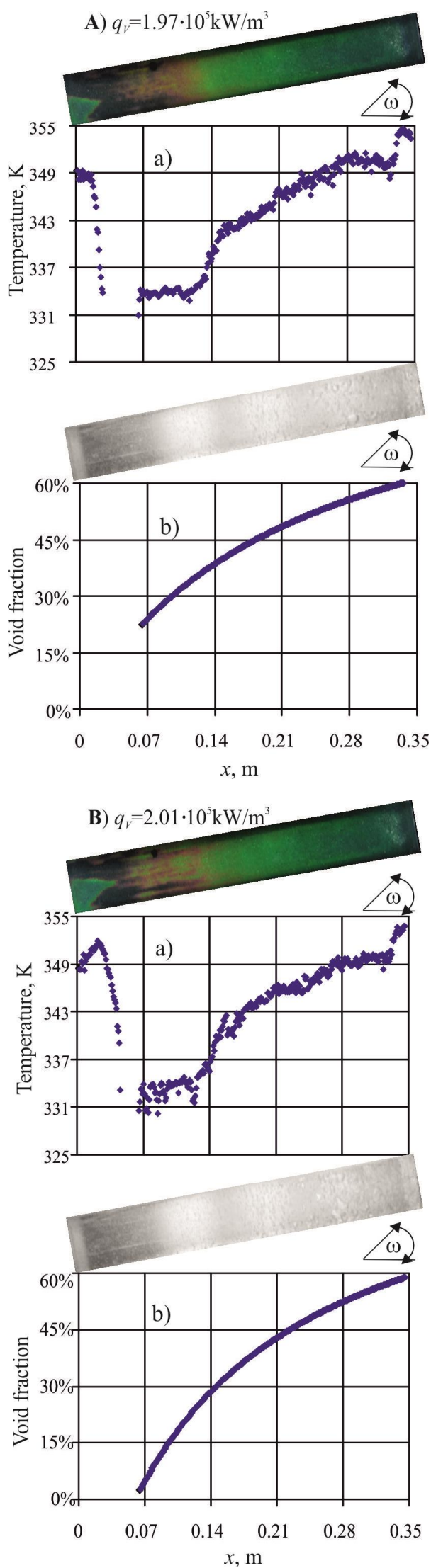

Fig.2. a) Foil surface temperature field obtained through liquid crystal thermography, b) flow structures and the corresponding void fraction determined from (9); refrigerant: fluorinert FC-72; volumetric heat flux supplied to the heating foil: A) $q_{V}=1.97 \cdot 10^{5} \mathrm{~kW} / \mathrm{m}^{3}$, B) $q_{V}=2.01 \cdot 10^{5} \mathrm{~kW} / \mathrm{m}^{3}$.

Experimental data from $[8,9]$.
The glass plate parameters were $\delta_{G}=0.006 \mathrm{~m}, H=0.35 \mathrm{~m}$, $\lambda_{G}=0.71 \mathrm{~W} /(\mathrm{mK})$ and the heating foil parameters were $\delta_{F}=1.016 \cdot 10^{4} \mathrm{~m}, \lambda_{F}=8.3 \mathrm{~W} /(\mathrm{mK})$ after $[8,9]$.

Figure 2a shows $2 \mathrm{D}$ temperature measurement results for the heating foil, obtained using the liquid crystal thermography method, and the corresponding flow structures. The liquid crystal measurements uncertainty is presented in [1] and the mean temperature measurement error is equal to $0.86 \mathrm{~K}$, [1]. The foil temperature distribution at the foil-glass interface, Fig.2a, was approximated using the polynomial of degree 5 . Figure $2 b$ shows the void fraction computed from formula (9).

For calculations, nine harmonic functions and six Trefftz functions $\theta_{n}(x, y)$ were used. The 2D approximation of the glass plate temperature was calculated in the first step, followed by the heating foil and then liquid in the last step. Figure 3 compares of the 2D temperature distributions for the glass plate $T_{G}$, heating foil, $T_{F}$ and liquid $T_{L}$, computed using the Trefftz method for data from Fig.2.

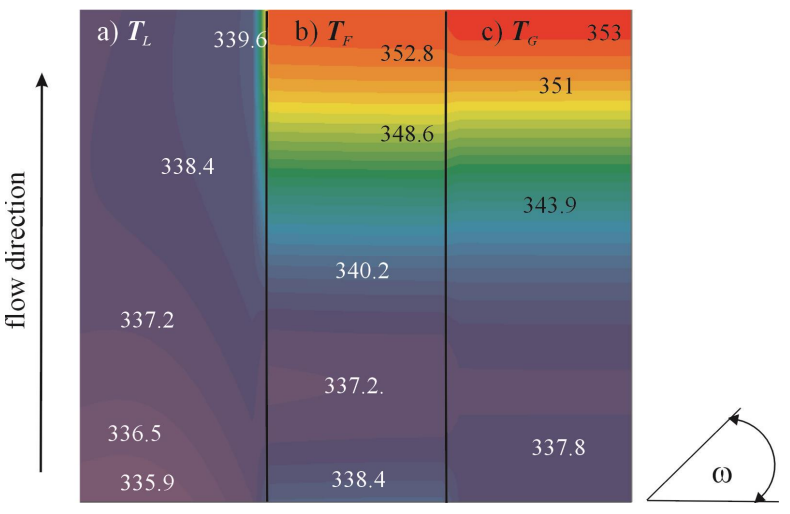

Fig. 3. Two-dimensional temperature fields determined by the Trefftz method for a)the glass plate $T_{G}$, b) the heating foil $T_{F}$, c) the liquid $T_{L}$. Additional data as in Fig. 2 for $q_{V}=1.97 \cdot 10^{5} \mathrm{~kW} / \mathrm{m}^{3}$.

To verify the accuracy to which the temperature approximations satisfied the adopted boundary conditions, the mean square error was defined as that for two arbitrary functions $f$ and $g$

$$
\varepsilon(f, g)=\|(f-g)\|
$$

It is apparent from Table 1 that the temperature approximations $T_{G}, T_{F}$ and $T_{L}$, determined using the Trefftz method, satisfied the boundary conditions to high accuracy. Condition (8) for the liquid temperature approximation, as the only one, was not satisfied with the accuracy comparable with other $\varepsilon$ values. This is due to the fact that the approximation of liquid temperature is determined by solving the inverse heat conduction problem as the second problem. The remaining boundary conditions, for which errors $\varepsilon$ have not been shown in Table 1, were satisfied to a relatively high accuracy. Analogous results were obtained for the data when $q_{V}=2.01 \cdot 10^{5} \mathrm{~kW} / \mathrm{m}^{3}$. 
Table.1 Mean square errors $\varepsilon$ between temperature approximations and selected boundary conditions: additional data as in Fig. 2 for $q_{V}=1.97 \cdot 10^{5} \mathrm{~kW} / \mathrm{m}^{3}$.

\begin{tabular}{|c|c|}
\hline $\begin{array}{c}\text { Errors } \varepsilon \text { for glass } \\
\text { temperature } \\
\text { approximation } T_{G}\end{array}$ & $\varepsilon\left(T_{G}\left(x_{m p}, \delta_{G}\right), T_{m p}\right)=1.29 \mathrm{~K}$ \\
\hline $\begin{array}{c}\text { Errors } \varepsilon \text { for foil } \\
\text { temperature } \\
\text { approximation } T_{F}\end{array}$ & $\varepsilon\left(T_{F}\left(x_{m p}, \delta_{G}\right), T_{m p}\right)=1.23 \mathrm{~K}$ \\
& $\left.\varepsilon\left(\lambda_{F} \frac{\partial T_{F}}{\partial y}, \lambda_{G} \frac{\partial T_{G}}{\partial y}\right)\right)=$ \\
& $=4.3 \cdot 10^{-4} \mathrm{~W} / \mathrm{m}^{2}$ \\
\hline $\begin{array}{c}\text { Errors } \varepsilon \text { for liquid } \\
\text { temperature } \\
\text { approximation } T_{L}\end{array}$ & $\varepsilon\left(T_{L}(0, y), T_{\text {in }}\right)=1.25 \mathrm{~K}$ \\
& $\varepsilon\left(T_{L}(H, y), T_{\text {out }}\right)=0.15 \mathrm{~K}$ \\
& $\varepsilon\left(\lambda_{L} \frac{\partial T_{L}}{\partial y}, \lambda_{F}(1-\varphi(x)) \frac{\partial T_{F}}{\partial y}\right)=$ \\
& $=385.15 \mathrm{~W} / \mathrm{m}^{2}$ \\
\hline
\end{tabular}

Two- dimensional temperature fields for FC-72 refrigerant computed by the Trefftz method for data from Fig. 2 are shows on Fig.4.

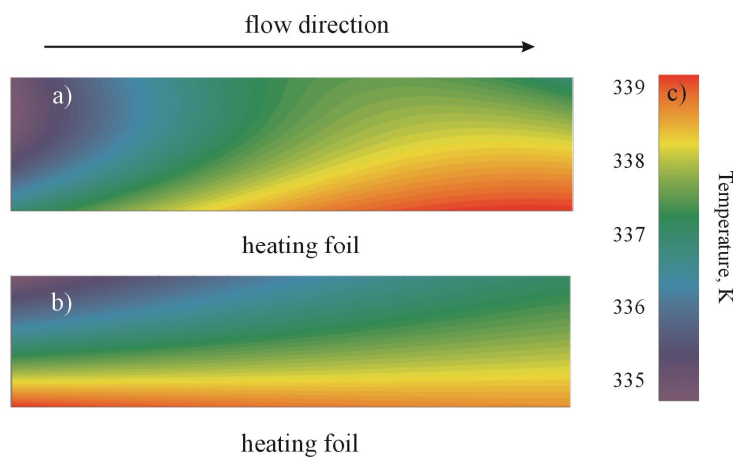

Fig. 4. Two-dimensional FC-72 temperature fields determined by the Trefftz method for a) $q_{V}=1.97 \cdot 10^{5} \mathrm{~kW} / \mathrm{m}^{3}$, b) $q_{V}=2.01 \cdot 10^{5} \mathrm{~kW} / \mathrm{m}^{3}$, c) scale. Additional data as in Fig. 2 .

Figure 4 shows the influence of the velocity profile and the minichannel tilt angle $\omega$ on the temperature distribution of the liquid, though for the higher value of $q_{V}=2.01 \cdot 10^{5} \mathrm{~kW} / \mathrm{m}^{3}$, this influence is weaker than for the lower value $q_{V}=1.97 \cdot 10^{5} \mathrm{~kW} / \mathrm{m}^{3}$.

The known temperature field of the liquid is used to determine the heat transfer coefficient $\alpha(x)$ at the foil liquid interface from (12). Figure 5 presents the heat transfer coefficient when the temperature was calculated using the Trefftz method. Confining our attention only to the thermal sublayer gave higher heat transfer coefficients compared to those obtained when the whole area was considered, Fig. 5. In the first section of the minichannel, where the share of the vapour phase was small and boiling was not occurring yet (see Fig. 2 b), an increase in the value of the heat transfer coefficient was observed. In the further section of the minichannel, when a two-phase flow occurred, the decrease in the value of the convective heat transfer coefficient resulted from an increase in the void fraction.
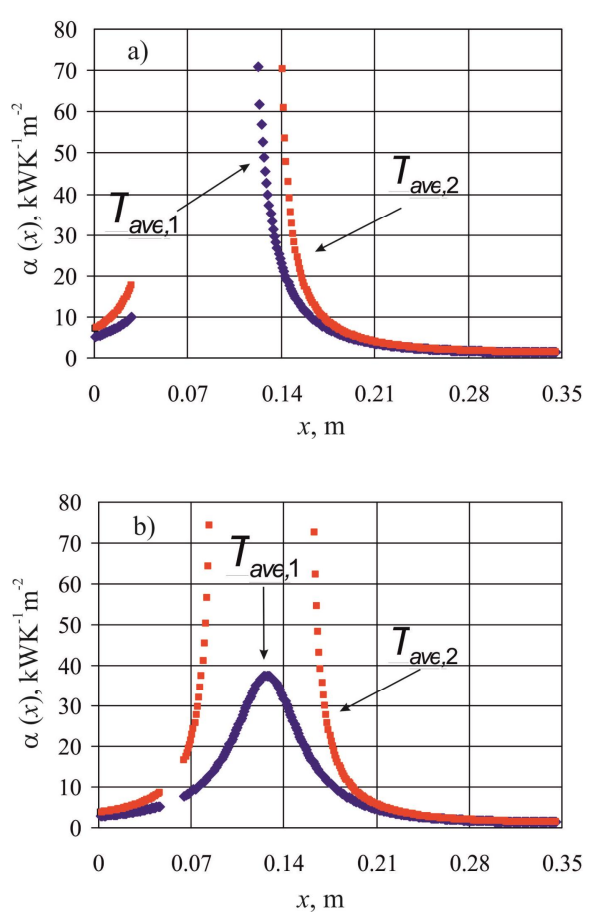

Fig.5. Heat transfer coefficients as a function of the minichannel height obtained using when the reference temperature is equal to $T_{a v e, 1}$ and $T_{a v e, 2}$ for:

a) $q_{V}=1.97 \cdot 10^{5} \mathrm{~kW} / \mathrm{m}^{3}$, b) $q_{V}=2.01 \cdot 10^{5} \mathrm{~kW} / \mathrm{m}^{3}$. Additional data as in Fig. 2 and saturation temperature: $338.5 \mathrm{~K} \div 340 \mathrm{~K}$.

\section{Conclusions}

In the paper the Trefftz method was applied for solving direct, inverse and conjugate inverse problems of a stationary heat conduction (including forced convection) in the solids and liquid. Harmonic functions and Trefftz functions for the energy equation, depending on the profile of the liquid velocity in the minichannel and the minichannel tilt angle, were used in the calculations. Both types of functions were used for the determination of approximate two-dimensional temperature distributions in three subsequent areas (glass pane, heating foil and flowing liquid), their gradients at the domain boundaries as well as the heat transfer coefficient at the heater-liquid interface.

It was observed that an increase in the share of vapour phase in a minichannel resulted in a decrease in the values of local heat transfer coefficients. The highest values of the heat transfer coefficient, of the order of $75 \mathrm{kWK}^{-1} \mathrm{~m}^{-2}$, were reached at the beginning of the saturated boiling region.

\section{References}

1. S. HoL̃ejowska, M. Piasecka, M.E. Poniewski, Int. J. Therm. Sci. 48, 1049-1059 (2009)

2. M. Piasecka, B. Maciejewska, Exp. Therm. Fluid Sci. 68, 459-467 (2015)

3. S. Hoúejowska, R. Kaniowski, M.E. Poniewski, Exp. Therm. Fluid Sci. 78, 18 Ï 29 (2016)

4. B. Maciejewska, M. Piasecka, Int. J. Heat Mass Transf. 107, 925 Ï 933 (2017) 
5. K. Grysa, A. MaciŃ Transf. 55, $7336 і ̈ 7340$ (2012)

6. M. Grabowski, S. HoL̈jowska, A. PawiGka, M.E. Poniewski, J. Wernik, Energies 11, 2057 (2018)

7. S. HoĹkjowska, J. Theor. App. Mech. 53, 969-980, (2015)

8. M. Piasecka, Int. J. Refrig. 56, 198-212 (2015)

9. M. Piasecka, Int. J. Heat Mass Transf. 81, 114-121 (2015)

10. M. Piasecka, B. Maciejewska, Exp. Therm. Fluid Sci. 44, 23-33 (2013)

11. A.S. Dalkilic, S. Laohalertdecha, S. Wongwises, Int. Comm. Heat Mass Transf. 36, 172-179 (2009)

12. G.P. Celata, S.K. Saha, G. Zummo, D. Dossevi, Int. J. Therm. Sci. 49, 1086-1094 (2010)

13. L.D. Landau, E.M. Lifshitz, Fluid Mechanics, (Pergamon Press, London, 1959)

14. R.K. Shah, A.L. London, Laminar flow forced convection in ducts: a source book for compact heat exchanger analytical data, (Academic Press, New York, 1978)

15. M. Piasecka, M.E. Poniewski, S. Hoüejowska, Int. J. Heat Fluid Flow 25 159-172 (2004)

16. S. HoL̃ejowska, R.Kaniowski, M.E. Poniewski, Int. J. Numer. Methods Heat \& Fluid Flow 24, 811-824 (2014)

17. S. HoL̃ejowska, L. HoL̃ejowski, Eng. Anal. Bound. Elem. (to be published) 